Varga, Krisztina (Budapest)

\title{
Wie und warum der Kitt gekaut wird
}

\author{
Überlegungen zur Kulturgebundenheit zweier Ausdrücke
}

\section{Einführung}

Grund, einstand, gittegylet - drei Wörter, die jeder ungarischer Muttersprachler kennt. Oder was es bedeutet, wenn ein Name mit kleinen Anfangsbuchstaben ins Vereinsbuch eingetragen wird... Es gehört zum ungarischen Kulturgut und wir wissen, dass sie in einem der beliebtesten ungarischen Romane vorkommen, im Jugendroman „A Pál utcai fiúk“.

Der Roman von Ferenc Molnár, „A Pál utcai fiúk“ wurde erstmals 1906 in der Jugendzeitschrift „Tanulók Lapja“ in Fortsetzungen veröffentlicht und eine Erstausgabe in Buchform erfolgte dann im Jahre 1907 beim Verlag Franklin Társulat. Das Buch gehört zu den meistgelesenen und beliebtesten Romanen in Ungarn und ist Pflichtlektüre in der fünften Klasse der Grundschule. Dies bedeutet also, dass Kinder schon als 10- bis 11-jährige mit dem Roman konfrontiert werden und es auch sehr gern lesen. Obwohl das Werk über 100 Jahre alt ist und die Geschichte die Realität am Anfang des 20. Jahrhundert widerspiegelt, hat das Buch nichts an seiner Popularität verloren; die seither etwas veraltete Sprache des ungarischen Originals sowie die zum Teil aus dem Deutschen in die ungarische Umgangssprache jener Epoche übernommenen Ausdrücke müssen für die Kinder und mit den Kindern interpretiert werden, um ihnen die Geschichte mit so wenig Verlust wie nur möglich nahezubringen. Dabei werden Ausdrücke wie gittegylet (ins Deutsche übersetzbar als ,Kittverein') sowie rágja a gittet (mit der ehemaligen Bedeutung: ,den Kitt kauen'), die den Untersuchungsgegenstand dieser Arbeit bilden, auch heute noch verwendet, allerdings nicht mehr im wortwörtlichen, sondern im übertragenen Sinn. ${ }^{1}$

Auch die internationale Popularität des Buches zeugt bis heute davon, dass es im Roman um immerwährende Lebenssituationen geht, die überall und jederzeit passieren könnten. Molnár beschreibt in seinem durch und durch Budapester Roman ohne Sentimentalität rührende Beispiele für Kameradschaft, Loyalität und Idealismus. Das Buch wurde in zahlreiche Sprachen übersetzt, in vielen Ländern (wie Italien, Brasilien, Polen und Japan) gilt es als obligatorische oder empfohlene Lektüre. $^{2}$

\footnotetext{
${ }^{1}$ Auf der Webseite des Petőfi Irodalmi Múzeums ist eine Liste von Wörtern aus der Jugendsprache der Jahrhundertwende (um 1900) zu finden: https://pim.hu/archivum/puf/object.999bcd84-cc66-4f71-bccd-ba11a223d384.ivy.html (Zugriff am 3. Januar 2022).

${ }^{2}$ Die Liste der Übersetzungen steht am Ende dieses Beitrags.
} 
Die Bedeutung und die Konnotation der Schlüsselwörter wie grund, einstand und gittegylet gehörten für die Kinder von damals zu ihrem Alltagsleben, daher war es für Molnár nicht weiter notwendig, eventuelle Erklärungen hinzuzufügen. Doch für die ungarischen Schüler von heute, die im 21. Jahrhundert leben, sind solche Orte, Gegenstände, Konzepte und Sachverhalte genauso fremd wie für die Leser des übersetzten Textes. Die meisten kulturspezifischen Konnotationen müssen den Lesern des Textes in der Zielsprache (ZS) ebenso zugänglich gemacht werden wie im Originaltext. In solchen Fällen befinden sich die Leser der Ausgangssprache (AS), die den Roman zum ersten Mal lesen, praktisch in derselben Position wie das zielsprachliche Publikum, legen Heltai / Fodor (2012) dar. Andererseits sind einige Begriffe im ungarischen Text gerade aus jenem Grund zu Kulturspezifika geworden, weil die meisten Ungarn diesen Roman gelesen haben: einstand und gittegylet können in sozialen und politischen Kontexten als Anspielungen verwendet werden. Die kulturspezifischen Konnotationen einiger Elemente hängen sogar mit der Tatsache zusammen, dass sie deutschen Ursprungs sind und von den AS-Lesern von heute auch als fremde Elemente empfunden werden. Für die Menschen im Budapest des frühen 20. Jahrhunderts, in dem viele Deutsch sprachen und viele deutsche Wörter nicht nur in die Umgangssprache, sondern auch in die Fachsprachen von damals übernommen wurden, waren diese fremden Elemente selbstverständlich.

Als Ausgangspunkt für die vorliegende Untersuchung dieser Elemente dienen folgende Ausgaben des Romans:

- Molnár, Ferenc (2020): A Pál utcai fiúk - A Gittegylet - bővített kiadás. Budapest: Móra Könyvkiadó. ${ }^{3}$

- Molnár, Ferenc (2002): Die Jungen der Paulstraße. Budapest: Corvina Kiadó.

- Molnár, Ferenc (2019): The Paul Street Boys. Budapest: Corvina Kiadó.

Der Beitrag setzt sich zum Ziel, die kulturelle Einbettung zweier kulturspezifischer Elemente darzustellen und einige Anhaltspunkte zur Geschichte der Begriffe nach dem Erscheinen des Romans zu geben. Anhand dieser Textbelege aus der Literatur soll die Kulturgebundenheit bestimmter Sachverhalte und Ausdrücke beschrieben werden: Als Ausgangssprache dient das Ungarische, die deutsche und die englische Sprache werden parallel als Zielsprachen betrachtet, wobei die Übersetzung ins Deutsche im Vordergrund stehen soll.

\footnotetext{
${ }^{3}$ Die neueste ungarische Ausgabe wurde 2019 mit dem Untertitel „A Gittegylet“ ergänzt.
} 
Im Rahmen dieser Arbeit sollen folgende Forschungsfragen beantwortet werden:

- Wie hat sich im Ungarischen die Bedeutung der Begriffe gittegylet bzw. rágja a gittet verändert?

- Auf welche Weise könnte diese Veränderung bei der Übertragung ins Deutsche bzw. ins Englische wiedergegeben werden?

Aus der Bedeutungsverschiebung ergibt sich, dass in dieser Arbeit die Ausdrücke sowie ihre (tatsächlich realisierten und möglichen) deutschen und englischen Äquivalenten auf zwei Ebenen beschrieben werden müssen: einerseits in Bezug auf ihre denotative Bedeutung (schließlich handelt es sich im Originaltext tatsächlich um einen Verein), andererseits auf den heutigen, idiomatisierten Gebrauch der Ausdrücke.

\section{Die Entstehungsgeschichte des Romans - der Weg zum geflügelten Wort}

Um die Bedeutung der beiden Begriffe besser verstehen zu können, soll an dieser Stelle kurz auf die Entstehungsgeschichte des Werks und die Umstände eingegangen werden.

Ferenc Molnár (1878-1952) wurde außerhalb Ungarns - vor allem in Österreich und nach seiner Emigration im Jahre 1940 in den USA - als Autor der Bühnenstücke „Liliom“, „Das Spiel im Schloss“ und „Der Schwan“ bekannt. In Ungarn ist er jedoch auch als Autor des Jugendromans „A Pál utca fiúk“ (1907) berühmt, den viele Lesergenerationen als ihr Lieblingsbuch aus der Kindheit in Erinnerung haben. Die Jungen der Paulstraße führen im Roman Krieg gegen eine andere Gruppe von Schuljungen um einen leeren Bauplatz, den grund, der für sie die Freiheit symbolisiert. In deutscher Sprache ist das Buch in der klassischen Übersetzung von Edmund Alkalay aus dem Jahr 1928 zu lesen. Die erste englische Übersetzung stammt von Louis Rittenberg, sie wurde 1927 veröffentlicht und später von George Szirtes für eine Neuveröffentlichung 1994 überarbeitet.

In Anlehnung an den Roman sind insgesamt sieben Verfilmungen entstanden, die bekannteste stammt aus dem Jahre 1969 und ist eine ungarisch-amerikanische Koproduktion (Regisseur: Zoltán Fábri). Der Film entstand 1968 und wurde Ende desselben Jahres in Ungarn uraufgeführt. In Deutschland kam der Film zwar nicht in die Kinos, er wurde aber am 1. November 1978 im ZDF erstmals ausgestrahlt. Der Film war 1969 sogar für den Oscar in der Kategorie „Bester fremdsprachiger Film“ nominiert. 2004 folgte dann auch eine deutschsprachige Verfilmung mit dem Titel „Die Jungen der Paulstraße“ Regisseur: Maurizio Zaccaro). 


\section{Die Fragen der Kultur und der Übersetzbarkeit}

Die Untersuchung von Realienlexemen oder kulturgebundener Lexik ${ }^{4}$ wirft interdisziplinäre Fragen auf, da an diesem Punkt die Linguistik auf die Forschungsfragen der Translatologie, sowie der interkulturellen Kommunikation trifft. Da sich meine Arbeit mit kulturell gebundenen Ausdrücken befasst, sowie der Realienbegriff vor allem in der Übersetzungswissenschaft verwendet wird, soll hier kurz auf die interkulturelle Kommunikation und ihren Zusammenhang mit der Translatologie eingegangen werden.

Der Kulturbegriff wird in der Alltagssprache sowie im wissenschaftlichen Sprachgebrauch mit unterschiedlicher Bedeutung verwendet. Während man im Alltag unter Kultur vor allem ihre Produkte versteht (Kunstwerke usw.), gebraucht die interkulturelle Kommunikation den Begriff im weiteren Sinne: Die Kultur ist eher ein gesellschaftlicher, menschlicher Schaffensprozess. Hidasi (2004: 11) formuliert dies folgendermaßen: „Eine jede Kultur schafft und vererbt ein gemeinsames Symbolsystem, mit dessen Hilfe und wodurch die Mitglieder einer Kultur miteinander in Kontakt treten, und ihr gemeinsames Dasein organisieren. " ${ }^{5}$ Gemäß dieser Kulturauffassung müssen wir zwischen sichtbaren (d. h. versachlichten) sowie wahrnehmbaren bzw. verborgenen Elementen unterscheiden. Die Letzteren, wie zum Beispiel Symbole, Normen, Werte, Erwartungen und Verhaltensformen sind die häufigsten und signifikantesten Komponenten der Kultur, deren Aneignung Sprachlerner, Geschäftsleute, Übersetzer sowie Dolmetscher gleichermaßen vor eine schwierige Aufgabe stellt. Da jedoch die Vertreter der ersten beiden Gruppen die problematischen Erscheinungen leichter umgehen können, stellen Metapher, Sprichwörter, Redewendungen und Mythen für die bewussten Sprachverwender der beiden letzten Berufsgruppen oft eine Herausforderung dar. Diese verborgenen Elemente gehören ebenfalls zur Kultur - Hidasi nennt sie Kultursymbole -, die Kultur tritt als ein gesellschaftliches Produkt auf, dessen System von Kenntnissen und Normen während der Sozialisation erworben wird. In einer neuen, ungewohnten Umgebung agiert diese Fähigkeit als ein Filter, da wir neue Erlebnisse, Eindrücke durch unser eigenes kulturelles System filtrieren und bearbeiten. Repräsentanten derselben Kultur verstehen sich auf Anhieb, während Mitglieder verschiedener Kulturen mehr Erklärung bedürfen. Dies muss von Übersetzern und Dolmetschern gleichermaßen bei ihrer Arbeit berücksichtigt werden.

Bei der Suche nach Wörtern und Ausdrücken mit einer kulturellen Bedeutungskomponente muss man als Übersetzer mit dem Kopf des ZS-Lesers denken und sogleich einen Vergleich zwischen der Kultur der AS und der der ZS

\footnotetext{
${ }^{4}$ Die Terminologie in der Fachliteratur ist nicht einheitlich, die Begriffe Realienlexem, kulturgebundener Ausdruck, kulturell gebundene Lexik, Realien usw. werden als Synonyme mit sehr ähnlicher, häufig jedoch nicht identischer Bedeutung verwendet. Ich verwende in erster Linie den Terminus Realienlexem s. Definition.

${ }^{5}$ Die Übersetzung der Textbelege und Zitate stammt von der Autorin dieser Arbeit.
} 
anstreben. Daraus folgt, dass Realienlexeme zugleich kultur- und sprachspezifisch, genauer gesagt, sprachpaarspezifisch sind. In der Relation zweier ferner Kulturen treten andere Gesichtspunkte in den Vordergrund als im Falle zweier verwandter Kulturen, wo ähnliche Sachverhalte dank der kleinen kulturellen Unterschiede nicht unbedingt als Realien angesehen werden.

Kujumäki (2004) macht auf einen weiteren Aspekt aufmerksam, wenn sie schreibt, dass die von ihr als Realienlexeme bezeichneten sprachlichen Zeichen für die Übersetzungswissenschaft in dem Falle relevant werden, wenn die Realien (d.h. die durch die Realienlexeme bezeichneten Kulturelemente) für eine bestimmte Kultur und/oder ein bestimmtes geografisches Gebiet charakteristisch sind, d. h. in anderen Kulturen/geografischen Gebieten nicht vorkommen. Sie fügt hinzu, aus dieser Auffassung ergäbe sich, dass die linguistisch ausgerichtete Translatologie die Sachverhalte als Spezifika definiert und deren Bezeichnungen, d. h. die Realienlexeme als Null-Lexeme oder auch Lakunen betrachtet (z. B. Koller 1992, Markstein 1998, Ertelt-Vieth / Denisova-Schmidt 2011). Papp (2004: 83) ergänzt noch, dass wegen dem fehlenden ZS-Äquivalent der Übersetzer das ausgeweitete semantische Feld, die damit verbundenen Assoziationen, die Atmosphäre, sowie den Stil des AS-Begriffes gleichzeitig in Erwägung ziehen muss. In der Kenntnis dieser trifft der Übersetzer dann am Ende des Übersetzungsprozesses eine subjektive Entscheidung.

Realienlexeme (Mujzer-Varga 2016) werden im Allgemeinen in Verbindung mit den Fragen der Übersetzbarkeit diskutiert, oftmals mit einer allgemeinen Gültigkeit, ohne die Bestimmung eines konkreten (sprachlichen oder vielmehr kulturellen) Rahmens, Kontextes, in dem die Kulturspezifik auftritt. Auch diese allgemeine Auffassung hat ihre Existenzberechtigung, angemessener wäre jedoch die Untersuchung des Realienbegriffs in Anbetracht konkreter Sprachpaare, da es problematisch ist, ohne Kontext von einem sprachlichen Zeichen zu entscheiden, ob es sich als Realienlexem verhält. Realienlexeme sind dementsprechend in jedem Fall im Zusammenhang zweier Sprachen (und Kulturen) - in der deutschen Fachliteratur heißt es sprachenpaarbezogen - zu interpretieren.

Daraus folgt ein weiterer Aspekt der Kulturgebundenheit: Ein Realienlexem muss nämlich meines Erachtens zwei Bedingungen entsprechen. Einerseits spreche ich von einem Realienlexem, wenn der Ausdruck in den Angehörigen einer sprachlich-kulturellen Gemeinschaft ähnliche Assoziationen hervorruft und/oder mit dem Alltagsleben dieser Gemeinschaft verbunden ist. Diese Funktion mag nicht jeder Vertreter der AS-Kultur gleich erkennen, da die sprachliche Äußerung zu seiner eigenen Welt, seiner Kultur, zu seinem Alltag gehört. Andererseits wird ein Realienlexem gerade aus dem Grunde zu einem Realienlexem, weil es für eine andere sprachliche Gemeinschaft keine zusätzliche Bedeutung trägt. Die Mitglieder einer fremden Gemeinschaft kennen eventuell die besagte sprachliche Äußerung nicht und sie erweckt in ihnen gerade deswegen gar keine oder unterschiedliche Assoziationen, in manchen Fällen wird sie sogar 
völlig missverstanden. Dies muss beim Übersetzen ebenfalls in Betracht gezogen werden (ebd.)

In dieser Arbeit soll nicht näher auf die Definierungsproblematik kulturspezifischer Bezeichnungen eingegangen werden. Im Folgenden wird folgende Definition als Ausgangspunkt dienen: Unter Realienlexemen verstehen wir alle sprachlichen Äußerungen, die für eine konkrete Gemeinschaft typisch sind, in deren Angehörigen dank ihrer gemeinsamen Hintergrundkenntnisse ähnliche Assoziationen hervorrufen, konnotative Bedeutung und emotionale Ladung haben. Aus der Definition geht hervor, dass die Betonung nicht auf der Unübersetzbarkeit der Realienlexeme liegt, sondern auf ihrer kulturellen Bestimmtheit.

\section{Gittegylet und rágja a gittet}

Die Begriffe haben sich so sehr in das Ungarische des 20.-21. Jahrhunderts eingenistet, dass Muttersprachler es in verschiedenen Situationen, insbesondere aber wenn es um Politik geht, problemlos verwenden können, ohne dabei über die Herkunft dieser Wörter nachzudenken. Aufgeführt werden hier einige Belege aus journalistischen Texten:

- Gittet rág ma Európa - Gittegylet lett ez a földrész! ${ }^{6}$ [Europa kaut heute Kitt - Dieser Kontinent ist zu einem Kittverein geworden!]

- Jogállamiság: rágják, mint a gittet [Rechtsstaatlichkeit: es wird wie am Kitt herumgekaut]

- Mert Amerika nemcsak az iraki háborúval kapcsolatos tehetetlenség miatt elégedetlen a világ legnagyobb gittegyletével. ${ }^{7}$ [Weil Amerika nicht nur wegen seiner Trägheit gegenüber dem Irak-Krieg mit dem größten Kittverein der Welt unzufrieden ist. $]^{8}$

- Rágjuk a gittet - A Pál utcai fiúk rágták, elsőként. Vagy csak ök vallották be elöször, hogy gittet rágni szükséges dolog, igaz, teljesen értelmetlen, de jó, szájról szájra adni meg de pláne. ${ }^{9}$ [Wir kauen den Kitt. Die Jungen in der Paulstraße kauten ihn zuerst. Oder sie waren die Ersten, die zugegeben haben, dass das Kauen von Kitt eine notwendige Sache ist, zwar völlig sinnlos, aber gut, und dass man ihn von Mund zu Mund gibt, ganz besonders.]

- Pass Lajos: Gittet rágni. ${ }^{10}$ [Am Kitt herumkauen]

${ }^{6}$ https://mandiner.hu/cikk/20170622_gagyor_peter_gittet_rag_ma_europa (Zugriff am 3. Januar 2022).

${ }^{7}$ https://ujszo.com/velemeny/ragjak-a-gittet (Zugriff am 3. Januar 2022).

${ }^{8}$ Kittverband und Kittverein werden von MS Word als unbekanntes/ungrammatisches Wort unterstrichen, gittegylet in der ungarischen Version von MS Word übrigens auch.

${ }^{9}$ https://kolozsvaros.com/2019/05/31/ragjuk-a-gittet/ (Zugriff am 3. Januar 2022).

${ }^{10}$ http://magyar-irodalom.elte.hu/ezredveg/2001/20013.html (Zugriff am 3. Januar 2022). 
- Olyan sok írás született már ebben a témában, egyáltalán nem akartam ezt a gittet tovább rágni. ${ }^{11}$ [Es wurde bereits so viel über dieses Thema geschrieben, dass ich diesen Kitt nicht mehr weiter kauen wollte.]

Das in dieser Arbeit zu untersuchende Wortpaar entspricht den Kriterien der geflügelten Worte (aus lexikologischer-phraseologischer Perspektive), andererseits haben wir es mit kulturspezifischen Elementen zu tun (unter dem Aspekt der Translatologie). Diese zwei Aspekte sollen nachfolgend erörtert werden.

Unter einem geflügelten Wort werden in der Alltagssprache die literarisch belegbaren, in den allgemeinen Sprachschatz des Volkes übergegangenen, allgemein geläufigen Redensarten verstanden. Bernát (1999) bemerkt in ihrer Arbeit zur Entstehung neuer Phraseologismen, dass Büchmann in seinem „Citatenschatz des Deutschen Volks“ ursprünglich keine Unterscheidung zwischen Zitaten und geflügelten Worten machen wollte oder konnte, und fügt hinzu, dass in der mehrfach überarbeiteten und ergänzten, aktualisierten 36. Auflage (1986) „geflügelte Worte nicht Zitate im engeren Sinne [sind], sondern solche, die, sozusagen losgelöst von ihrem Autor und ihrem Zusammenhang, in übertragenem Sinne angewendet werden und dabei in den alltäglichen Sprachschatz eingehen, wobei sie auch ihren Wortlaut ändern können.“ (Büchmann 1986: VIII). In der Einleitung vom Band 12 des Dudens („Zitate und Aussprüche“; 1993: 13) wird auch darauf hingewiesen, dass heute weniger aus Werken zitiert wird. Sehr häufig sind die Titel von Spielfilmen and Fernsehserien die Lieferanten moderner Zitate, und nicht mehr das literarische Kulturgut, dass seinen Weg in die Sprache der Menschen findet.

Der zweite Aspekt bezieht sich auf die Realienforschung, die wichtige Hinweise über den kulturellen Hintergrund sowie die zur Verfügung stehenden Übersetzungsverfahren liefert. Die breite Realienauffassung von Drahota-Szabó (2013: 24f) weist zahlreiche Übereinstimmungen mit meiner Definition auf (vgl. Kapitel 2). Unter Realien versteht sie neben den Bezeichnungen der kulturspezifischen Gegenstände und Begriffe (z.B. Speisen, Trachten, Währungen, Feste, Möbelstücke, Verkehrsmittel), der politischgesellschaftlichen Institutionen und Begriffe (z.B. Bezeichnungen im Zusammenhang mit der Staatsverwaltung und Nachrichtenübermittlung, mit dem Gesundheitswesen und Handel usw.; Bezeichnung von sozialen Schichten) und Eigennamen (Personennamen, geographischen Namen usw.) auch diejenigen sprachlichen Elemente, die mit der jeweiligen Kultur aufs Engste verknüpft sind (z.B. Berufsbezeichnungen). Was allerdings im Gegensatz zu meiner ebenfalls breiten Auffassung steht, ist, dass Drahota-Szabó auch feste Wendungen im breitesten Sinne, also Phraseologismen, d.h. Redewendungen, Sprichwörter,

\footnotetext{
${ }^{11} \mathrm{http}: / /$ szentjobbikriszta.hu/szponzoralt-tartalom-reklam-promocio-mint-nehezen-emeszhetofinomsagok/ (Zugriff am 15. April 2021).
} 
geflügelte Worte, Titel von/Zitate aus literarischen Werken, politische Losungen, Ausdrücke der verbalen Aggressivität wie Beschimpfungen und Flüchen zu den kulturellen Realien zählt.

\subsection{Die denotative Bedeutung der Ausdrücke gittegylet und rágja a gittet}

Einige der Mitglieder aus der Bande der Paulstraße haben sich etwas abgesondert und als Zeichen ihrer Selbständigkeit (und um sich ein wenig wichtig zu tun) den sog. Kittverein (im Original: gittegylet) gegründet.

Das Material Kitt ist aus dem Bauwesen bekannt und wird in ihrer primären Bedeutung zum Kleben und Abdichten von Fenstern verwendet:

- Laut „Duden“: Kitt, der: zum Kleben, Dichten o. Ä. verwendete, knetbare oder zähflüssige Masse, die an der Luft erhärtet ${ }^{12}$ bzw.:

- Laut „DWDS“: Kitt, der: teigartiger, klebriger Stoff, der an der Luft erhärtet und zum Kleben, Binden, Dichten oder zum Ausfüllen von Fugen verwendet wird. ${ }^{13}$

Der Verein im Roman wurde nach dem Vorbild eines Erwachsenenvereins gegründet, mit einem Präsidenten (Boka), und in diesem Verein hat jeder eine „Funktion“, doch hier geht es um eine Vereinigung, die keinen anderen Zweck hat als das Vereinigen selbst. Sie kauen am Kitt herum, und das ist das alleinige Ziel des Vereins. Einige der Verteidiger des „Grunds“, dieses unbebauten Grundstücks, der für die Kinder ihr Heimatland darstellt, kümmern sich um den Kitt mit heldenhafter Ausdauer. Der Kitt wird von Mund zu Mund gegeben und gekaut, um ihn feucht zu halten. Allmählich hat der Begriff gittrágás ,Kittkauen“ in der ungarischen Sprache eine Nebenbedeutung erhalten, die sich auf die Ausführung belang- oder zweckloser Handlungen bezieht.

Wir werden nie erfahren, warum Ferenc Molnár den Verein der Kinder und Heranwachsenden (Boka wird von den Kleineren für einen richtigen Mann gehalten, der den Kittverein zum Bedauern der Vereinsmitglieder nicht wirklich ernst nimmt) in die heldenhafte und tragische Geschichte von Ernő Nemecsek aufgenommen hat, aber die Existenzberechtigung des Vereins wird mehrmals angezweifelt. Wenn wir andererseits das historische Zeitalter betrachten, in dem der Roman entstanden ist, können wir davon ausgehen, dass der Autor nicht versehentlich die Arbeitsweise dieses Vereins darin darstellt. Die Mitglieder sind Kinder und da das Ganze nur ein Spiel ist, kann es von der Außenwelt, wie den Lehrern, aber auch von den anderen Kindern außerhalb des Vereins nicht ernst genommen werden. Davon zeugt auch die Benennung ihrer

\footnotetext{
12 https://www.duden.de/rechtschreibung/Kitt (Zugriff am 3. Januar 2022).

${ }^{13}$ https://www.dwds.de/wb/Kitt (Zugriff am 3. Januar 2022).
} 
„Interessengemeinschaft“ im Originaltext: egylet ist umgeben von einem Hauch Seriosität; nur diejenigen können Mitglieder werden, die die Sache auch ernst nehmen, die bereit sind, Opfer zu erbringen. Wird ein solches Wort hingegen mit einer klebrigen Masse wie gitt kombiniert, verliert das Kompositum, das auch im Ungarischen eine Gelegenheitsbildung ist, sofort an ihrer Respektabilität.

Auf der wortwörtlichen, denotativen Ebene lassen sich die Begriffe mit Leichtigkeit ins Deutsche übertragen: als Kittverein bzw. am Kitt herumkauen. Das Übersetzungsverfahren, das hier beim ersten Wort in Anspruch genommen wird, wird in der Fachliteratur als Direkte Übersetzung (oder auch wörtliche Übersetzung oder Lehnübersetzung) bezeichnet, wobei jedes einzelne Element aus der Ausgangssprache in die Zielsprache übertragen wird: gitt+egylet $=>$ Kitt+Verein. Das Wort verfügt im Ungarischen übrigens über zwei Schreibweisen, gitt wird ausschließlich in Bezug auf Fensterverdichtung gebraucht (im Gegensatz zu kitt ,Leim, Klebstoff'). In Anlehnung an Pedersen verstehe ich unter Direkter Übersetzung (vgl. direct translation) ein Übersetzungsverfahren, bei dem der semantische Inhalt des AS-Ausdrucks unverändert bleibt, es wird jedoch kein Versuch unternommen, Konnotationen zu übertragen oder dem ZS-Leser auf irgendeine Weise das Verständnis zu erleichtern.

Das Wort Verein lässt sich aufgrund seiner denotativen Bedeutung am besten mit egylet übersetzen. Ein möglicher Übersetzungsverlust kann darauf zurückgeführt werden, dass egylet im Ungarischen als ,veraltet/veraltend“ empfunden wird. Eine kurze Recherche in der Online-Version des „Dudens“ ergibt insgesamt 36 Synonyme zu Verein, ${ }^{14}$ neben der Angabe der Stilebenen, der regionalen Zuordnung sowie der Zuordnung zu Fach- oder Sondersprachen fehlt jedoch die zeitliche Zuordnung des Wortes. Der von den ungarischen Muttersprachlern als abwertend-pejorativ empfundene Charakter des zusammengesetzten Wortes kann ebenfalls nicht mithilfe der im „Duden“ aufgeführten Synonyme wiedergegeben werden. Im „Digitalen Wörterbuch der deutschen Sprache“ wird allerdings neben der primären Bedeutung (,Zusammenschluss, Vereinigung von Personen zu dem Zweck, sich auf einem bestimmten Gebiet gemeinsam zu betätigen, gemeinsame Interessen zu pflegen') auch die Nebenbedeutung erwähnt ([umgangssprachlich, spöttisch, übertragen], Gruppe von Menschen, an deren Verhalten Kritik geübt wird'). ${ }^{15}$

Der deutschsprachige Leser (und wir sprechen ja hier in erster Linie von Kindern), der beim Lesen des deutschen Textes zum ersten Mal mit dem zusammengesetzten Wort Kittverein konfrontiert wird, kann durchaus Verständnisprobleme haben. Schließlich handelt es sich hierbei um eine okkasionelle Wortbildung, d.h. eine spontan entstandene, kontextabhängige Gelegenheitsbildung, deren Bedeutung aus den Bestandteilen herleitbar ist. Zum

\footnotetext{
${ }^{14}$ https://www.duden.de/synonyme/Verein (Zugriff am 3. Januar 2022).

${ }^{15}$ https://www.dwds.de/wb/Verein (Zugriff am 3. Januar 2022).
} 
Glück können in einem Roman die Umstände genau erklärt werden, und das Wort wird eingeführt ohne Missverständnisse zu verursachen. Ähnlich verhält es sich auch mit der englischen Entsprechung, the Putty Club, einer Lehnübersetzung aus dem Ungarischen.

Die Verbkonstruktion rágja a gittet kann mit einem Akkusativobjekt übernommen werden: rágja a gittet $=>$ (wortwörtlich:) kaut den Kitt. Allerdings soll hier der Vorschlag gemacht werden, dass gerade wegen der übertragenen Bedeutung des Wortes der iterative Charakter beibehalten werden soll: am Kitt herumkauen, das Wort erinnert meines Erachtens absichtlich an das Wiederkauen von Kühen. Ins Englische lässt sich die Wortverbindung am einfachsten mithilfe der Direkten Übersetzung als to chew the putty übersetzen.

\subsection{Die übertragene Bedeutung von gittegylet und rágja a gittet}

Spannend in diesem Roman ist auch die Symbolik des Kitts. Der Kitt ist schließlich ein Symbol der Zusammengehörigkeit, wie auch im folgenden Beispiel: „ein Kind ist kein Kitt (Bindemittel; etwas, was den Zusammenhalt erhält (für eine Ehe) “. ${ }^{16}$ Im Roman ist es dieses klebrige Material, das die Mitglieder des Kittvereins zusammenhalten soll, ohne dieses Material würde der Verein gar nicht existieren. Der Kitt hält den Verein zusammen, gibt den Kindern ein Ziel. Nachdem sie jedoch aufgeflogen sind, haben sich die Jungs gegeneinander gewandt, und der Verein verlor seinen Zweck - ohne den Kitt waren sie nichts!

Die Beschreibung der damaligen soziokulturellen und -linguistischen Umstände versorgt den Übersetzer und somit auch die Leser mit den notwendigen Informationen, die für das Verständnis der Begriffe und ihre Übersetzung unerlässlich sind. Dies hilft dem Übersetzer dabei, mithilfe des entsprechenden Übersetzungsverfahrens eine gute Lösung zu finden, die in den Lesern der Zielsprache die gleichen oder ähnliche Konnotationen, Assoziationen und Gefühle hervorruft wie das Original in den Lesern der Ausgangskultur und Ausgangssprache. Denn dies ist schließlich das Ziel einer guten literarischen Übersetzung. Kittverein und the Putty Club haben somit ihren Weg in die Übersetzung des Romans gefunden.

Es ist gar nicht so leicht, in Nachschlagewerken eine geeignete Definition für die übertragene Bedeutung des Originalwortes zu finden:

\footnotetext{
${ }^{16}$ https://www.duden.de/rechtschreibung/Kitt (Zugriff am 3. Januar 2022).
} 
- gittegylet [t-e] főnév (gúnyos)

Fölöslegesen alakult, jelentéktelen ügyekkel foglalkozó kis egyesület, szervezet; (rosszalló) Fontoskodó, de komoly ügyintézésre képtelen szervezet, intézmény. ${ }^{17}$

- A gittegylet gúnyos kifejezés olyan társaságra, egyesületre stb., amelynek látóköre, tevékenysége nem terjed túl tagjainak érdeklődésén (mely mások számára jelentéktelen. ${ }^{18}$

- gittegylet: Egy pár emberből álló társaság, amely lényegtelen ügyekben ülésezik. $^{19}$

Ein gittegylet ist in der heutigen ungarischen Umgangssprache also ein kleiner Verein und eine kleine Organisation, die sich mit unbedeutenden Angelegenheiten befasst, die eigentlich unnötigerweise gegründet wurde. Das Wort hat eindeutig mit Wichtigtuerei zu tun, diese Bedeutungskomponente ist allen ungarischen Muttersprachlern bekannt. Die Aufrechterhaltung dieser Gruppe ist das einzige Ziel dieser Gruppe. Diese Wichtigtuerei oder dieses Sichaufspielen macht sich auch dadurch bemerkbar, dass die Jungs alle darauf erpicht waren, eine wichtige Position oder Funktion innerhalb des Vereins einzunehmen, doch als es darum ging, wirklich etwas zu tun, meldete sich keiner freiwillig. Es gab aber Regeln, die zu befolgen waren.

Unter Punkt 4.1 wurden eventuelle Möglichkeiten der Übertragung vorgelegt (Kittverein bzw. am Kitt herumkauen; the Putty Club bzw. to chew the putty), wobei die angewandten Übersetzungsverfahren als Direkte Übersetzung einzuordnen sind. Im Gegensatz dazu kann bei der Übersetzung der übertragenen Bedeutung in erster Linie das Übersetzungsverfahren Adaptation angewendet werden. Unter Adaptation im engeren Sinne versteht Koller „die Ersetzung des mit einem AS-Ausdruck erfaßten Sachverhalts durch einen Sachverhalt, der im kommunikativen Zusammenhang der ZS eine vergleichbare Funktion bzw. einen vergleichbaren Stellenwert hat“ (Koller 1992: 234). Wenn wir die Umstände und den Kontext in Betracht ziehen, in denen dieser Begriff verwendet werden kann, sollten wir nach einer Entsprechung mit einer ähnlichen Funktion suchen, wenn möglich in literarischen Werken o.Ä. wie z.B. The Royal Society for Putting Things on top of Other Things in den Sketchen der Monty Pythons oder The Pickwick Club bei Dickens. Weitere Vorschläge sind all talk, no action group

${ }^{17}$ http://ertelmezo.oszk.hu/kereses.php?kereses=gittegylet; [Überflüssige kleine Vereinigung oder Organisation, die sich mit unbedeutenden Angelegenheiten befasst; (missbilligend) eine sich wichtigtuende Organisation oder Institution, ohne die Fähigkeit zum Verwalten] Zugriff am 5. Dezember 2021).

${ }^{18}$ https://hu.wikipedia.org/wiki/Gittegylet, [Ein Kittverein ist ein spöttischer Begriff für ein Unternehmen, einen Verein usw., deren Blickfeld und Aktivitäten nicht über das (für Andere unbedeutende) Interesse seiner Mitglieder hinausgehen.] Zugriff am 15. Februar 2021).

${ }^{19}$ https://hu.wiktionary.org/wiki/gittegylet [Eine Gemeinschaft aus wenigen Leuten, die wegen unbedeutenden Kleinigkeiten tagt.] Zugriff am 15. Februar 2021). 
'nur-Gerede-keine-Aktion-Gruppe' oder bootless club / company , nutzloser Club / Gemeinschaft'. Die beiden Letzteren betonen die Ziellosigkeit oder Sinnlosigkeit des Vereins. ${ }^{20}$ Neben der Adaptation gibt es auch weitere Übersetzungsverfahren, die ein Übersetzer hier anwenden kann. Bei literarischen Texten, insbesondere bei Kinder- und Jugendliteratur muss besonders darauf geachtet werden, dass sich die Texte leicht und fließend lesen lassen, stilistische Kriterien müssen ebenfalls in den Vordergrund gerückt werden. Es gehört zu den Aufgaben der Übersetzer, den ZS-Text so gut wie möglich zu interpretieren und damit zu erreichen, dass ZS-Leser so wenig Energie und Zeit investieren müssen, um z.B. kulturspezifische Elemente verstehen zu können.

Die AS-Ausdrücke gittegylet und rágja a gittet sind im Ungarischen Gelegenheitsbildungen, deshalb werden sie in einsprachigen ungarischen Wörterbüchern kaum aufgeführt, weder in ihrer ursprünglichen noch in ihrer übertragenen Bedeutung. Eine Ausnahme bildet das Ungarisch-Deutsche Großwörterbuch von Halász / Földes / Uzonyi, in dem gittegylet als ,nutzloser Verein` angegeben ist (1998: 547).

$\mathrm{Zu}$ diesem Begriffsfeld passt noch ein weiterer Ausdruck, nämlich die Vereinsmeierei, ein Begriff aus der Psychologie, die sich pejorativ auf Menschen bezieht, „denen die Mitgliedschaft und Mitarbeit in einem oder mehreren Vereinen außerordentlich wichtig ist. Abgeleitet hiervon bezeichnet Vereinsmeierei eine Haltung, in der der Betätigung in einem Verein und dem Wirken von Vereinen übertriebener Wert beigemessen wird. Oft wird damit auch eine Bürokratie innerhalb eines Vereins kritisiert, wenn zu viel Wert auf Formalien anstatt auf die inhaltliche Vereinsarbeit gemäß der Satzung (CH: Statuten) des Vereins gelegt wird“. ${ }^{21}$ Laut Duden ist dieses Wort umgangssprachlich abwertend gebraucht ${ }^{22}$, und stellt eine Haltung dar, die sich durch ,übertriebenes Wichtignehmen der Betätigung in einem oder mehreren Vereinen" manifestiert.

Wie unter 4.1 erörtert wurde, kann die ursprüngliche Bedeutung der Verbalkonstruktion mit den Kitt kauen/am Kitt kauen wiedergegeben werden. Ihre übertragene Bedeutung lässt mithilfe der stilistisch neutralen Wortverbindung jemand vollzieht unbedeutende oder nutzlose Handlungen definieren. Als sehr umgangssprachlich-pejorative Entsprechungen gelten folgende Wendungen, die die abstrakte Bedeutung gut abdecken: leere/hohle Phrasen dreschen, leeres Stroh dreschen, herumlabern, sich das Maul zerreißen, sprechen um das Sprechen willen bzw. Reden um das Reden willen, eventuell in Frage kommen auch die folgenden umgangssprachlichen Ausdrücke: Scheiße dreschen oder Furz schälen, leider konnten dafür keine verlässlichen Quellen gefunden werden. Im Englischen

${ }^{20}$ Der Begriff gittegylet ist übrigens so fest in der ungarischen Sprache verankert, dass z.B. der Film „The Boys Club“ aus dem Jahre 1996 unter dem Titel „Gittegylet“ in Ungarn gespielt wurde.

${ }^{21}$ https://de.wikipedia.org/wiki/Vereinsmeierei (Zugriff am 3. Januar 2022).

${ }^{22}$ https://www.duden.de/rechtschreibung/Vereinsmeierei (Zugriff am 3. Januar 2022). 
eignen sich die Verben to blabber, to bloviate. Falls die große Menge des Redeflusses im Vordergrund steht, spricht man im Englischen über babble, idle talk, senseless prattle, gabble, twaddle oder sogar logorrhea.

Im Ungarischen wird die Wortverbindung rágja a gittet auch häufig als zusammengesetztes Wort gittrágás verwendet, das im Deutschen als dummes / sinnloses Geschwätz / Gerede / Gelaber / Gefasel, Phrasendrescherei nichtssagendes Geschwätz, Geplapper, Gewäsch, Gesülze, leeres Wortgeklapper, im Englischen als talking for the sake of talking bzw. talking for talking's sake, purposeless / futile conversation wiedergegeben werden kann.

Das Originalwort kann neben der Bedeutung ,sinnloses Geschwätz' auch eine Nebenbedeutung wie sich aufspielen, Rabatz machen, viel Aufhebens um etwas machen haben.

\section{Literatur}

Molnár, Ferenc (2020): A Pál utcai fiúk - A Gittegylet. Bővített kiadás. Budapest: Móra Könyvkiadó.

Molnár, Ferenc (2002): Die Jungen der Paulstrasse. Budapest: Corvina Kiadó. (Übersetzt von Edmund Alkalay)

Molnár, Ferenc (2019): The Paul Street Boys. Budapest: Corvina Kiadó. (Übersetzt von Louis Rittenberg)

Bernáth, Csilla (1999): Zur Entstehung neuer Phraseologismen - Filmtitel auf dem Weg zum geflügelten Wort. in: Bassola, Peter / Oberwagner, Christian / Schnieders, Guido Schnittstelle (1999): Deutsch. Linguistische Studien aus Szeged. Festschrift für Pavica Mrazovic. Szeged: Grimm Könyvkiadó. S. 169-173

Büchmann, Georg (1986): Geflügelte Worte. 36. Auflage. Bearbeitet von W. Hofmann. Frankfurt, Berlin.

Drahota-Szabó, Erzsébet (2013): Realien - Intertextualität - Übersetzung. Beiträge zur Fremdsprachenvermittlung, Sonderheft 19.

DUDEN (1993): Zitate and Aussprüche. Duden, Band 12, Mannheim.

Ertelt-Vieth, Astrid / Denisova-Schmidt, Elena (2011): Lakunen-Analyse zur empirischen Forschung interkultureller Kommunikation - Methodologie. In: Roesch O (ed) Osteuropa interkulturelle, interlinguale und kulturvergleichende Studien. Berlin: News \& Media, pp. 170-187.

Fodor, Balázs / Heltai, Pál (2012): Culture-specific items in translation In: Navracsics, Judit / Szabó, Dániel (Hgg.) (2012): Mentális folyamatok a nyelvi feldolgozásban. Budapest: Tinta Könyvkiadó. 306-318.

Halász, Előd / Földes, Csaba / Uzonyi Pál (Hgg.) (1998): Magyar-német Nagyszótár. Budapest: Akadémiai Kiadó.

Hidasi, Judit (2004): Interkulturális kommunikáció. Budapest: Scholar Kiadó.

Koller, Werner (1992): Einführung in die Übersetzungswissenschaft. Heidelberg: Quelle\&Meyer.

Kujumäki, Pekka (2004): Übersetzung von Realienbezeichnungen in literarischen Texten. In: Kittel, Harald et al. (2004) Übersetzung - Translation - Traduction. Berlin-New York: Walter de Gruyter. 920-925. 
Mujzer-Varga, K. (2016): Verfremdung oder Adaptierung: Realienlexeme in den Übertragungen der Minutennovellen von István Örkény. Hamburg: Verlag Dr. Kovac.

Markstein, Elisabeth (1998): Realia. In: Snell-Hornby, M. et al. (Hgg.) Handbuch Translation. Tübingen: Stauffenburg Verlag.

Papp Nándor (2004): Terminológia és reáliák. In: Dróth J. (Hgg.) Szaknyelv és szakfordítás. Gödöllő: Szent István Egyetem, 79-90.

Pedersen, Jan (2007): “How is culture rendered in subtitles?”. In: Nauert, S. (Hgg.) Challenges of Multidimensional Translation. Proceedings of the Marie Curie Euroconferences MuTra: Challenges of Multidimensional Translation - Saarbrücken 2-6 May 2005. [www.euroconferences.info/proceedings/2005_Proceedings/2005_proceedings.html, Zugriff am 22. Februar 2021] 


\section{Anhang - Übersetzungen des Werks}

(1) Djemtë e rrugës Pal sowie Çunat e rruges Pal (Albanisch)

(2) The Paul Street Boys sowie No Greater Glory (Englisch)

(3) Момчетата от Павловата улица sowie Момчетата от улица Пал [Momcsetata ot ulica Pal] (Bulgarisch)

(4) Chlapci z Pavelské ulice oder Hoši z Pavelské ulice (Tschechisch)

(5) La knaboj de Paŭlo-strato (Esperanto)

(6) Pál-tänava poisid (Estnisch)

(7) Koulupoikia (kirja Pál-kadun pojista) (Finnisch)

(8) Les garçons de la rue Pál sowie Les gars de la rue Paul (Französisch)

(9) Paliskuzeli bizebi (Grusisch)

(10) מחנים Máhánájim (Hebräisch)

(11) De jongens van de Pálstraat (Niederländisch)

(12) Junaci Pavlove Ulice és Dječaci Pavlove ulice (Kroatisch)

(13) パール街の少年たち Pārugai no shōnentachi (Japanisch)

(14) 팔 거리의 아이들 Phal korii aidul (Koreanisch)

(15) Chłopcy z Placu Broni (Polnisch)

(16) Palo gatvès berniūkščiai (Litauisch)

(17) Decata od Pavlovata ulica (Mazedonisch)

(18) Die Jungen der Paulstraße (Deutsch)

(19) Kampen om løkka (Norwegisch)

(20) I ragazzi della via Pal sowie I ragazzi della via Paal (Italienisch)

(21) Мальчишки с улицы Пала (Russisch)

(22) Pal csampu teghake (Armenisch)

(23) Os meninos da rua Paulo (Portugiesisch)

(24) Băieţii din strada Pal (Rumänisch)

(25) Los chicos de la calle Paul (Spanisch)

(26) På liv och död (Schwedisch)

(27) Junaci Pavlove Ulice sowie Дечаци Павлове улице / Dečaci Pavlove ulice (Serbokroatisch)

(28) Chlapci z Pavlovskej ulice (Slowakisch)

(29) Dečki Pavlove ulice (Slowenisch)

(30) Pal Sokağı Çocukları sowie Pal Sokağının Çocukları (Türkisch)

(31) Хлопці з вулиці Пала [Hlopci z vulüci Pala] (Ukrainisch)

(32) Những cậu con trai phố Pál és Những cậu con trai phố Pan (Vietnamesisch) 